\title{
Reconstruction of the eddy current profile on the vacuum vessel in a nuclear fusion device using only external magnetic sensor signals
}

\author{
M. Itagaki ${ }^{1}$, A. Sanpei ${ }^{2}$, S. Masamune ${ }^{2} \&$ K. Watanabe ${ }^{3}$ \\ ${ }^{1}$ Faculty of Engineering, Hokkaido University, Japan \\ ${ }^{2}$ Kyoto Institute of Technology, Japan \\ ${ }^{3}$ National Institute for Fusion Science, Japan
}

\begin{abstract}
In a reversed field pinch device, the ordinary Cauchy-condition surface (CCS) method cannot reconstruct the magnetic flux profile accurately due to the strong eddy current flow on the shell (vacuum vessel). Boundary integrals of the eddy current density along the shell are added to the original CCS method formulation. The eddy current profile is unknown in advance but identified using only the signals of magnetic sensors located outside the plasma. As the sensors are closely adjacent to the shell, the near singular boundary integrals along the shell should be accurately evaluated. This singularity can be damped out with the algorithm based on the distance function proposed by Ma and Kamiya. The modified truncated singular value decomposition technique of Hansen et al. is an effective way to solve an ill-conditioned matrix equation when a large number of nodal points exist on the shell. The capability of the new method is demonstrated for a test problem modelling the RELAX device.

Keywords: Cauchy-condition surface, eddy current, magnetic sensor signal, singular integral, modified truncated singular value decomposition technique.
\end{abstract}

\section{Introduction}

In a nuclear fusion device, the magnetic field or flux profile outside the plasma and hence the plasma boundary shape are highly important for operating control and diagnosis. Such information should be deduced from signals of magnetic sensors located outside the plasma, since the direct measurement of physical quantities inside the plasma is usually difficult. The Cauchy condition surface 
(CCS) method $[1,2]$ is one such idea for reconstructing the flux distributions around the plasma boundary. The method has already been established for operating control of JT-60U, a tokamak-type device. In the method, however, the eddy current generated on the vacuum vessel was neglected. In a reverse field pinch (RFP) device such as the RELAX [3] at Kyoto Institute of Technology, a strong eddy current is generated on the shell (vacuum vessel) wall, which is closely related to the local magneto-hydrodynamic (MHD) equilibrium.

In the present paper the authors propose an advanced CCS method where the eddy current term is given by a boundary integral along the shell in the poloidal direction. The eddy current profile is not given in advance but completely unknown before one starts the analysis. One solves the Cauchy conditions and the eddy current profile simultaneously. The authors introduce two ideas to overcome the numerical difficulties encountered in this inverse problem. One is an accurate boundary integral scheme to damp out the near singularity occurring at the sensor position very close to the shell. The other is the modified truncated singular value decomposition (MTSVD) technique to solve an ill-conditioned matrix equation when a large number of nodal points exist on the shell.

\section{Problem specifications}

One here considers a problem to model a limiter configuration of the RELAX device [3], as an example of a reversed field pinch device. The shell (vacuum vessel) is regarded as axisymmetric in the toroidal direction and its cross section is a circle with radius $0.25 \mathrm{~m}$, which is centred at $(r, z)=(0.51 \mathrm{~m}, 0.51 \mathrm{~m})$ as shown in Figure 1. One assumes that a 'limiter' having a length of $1 \mathrm{~cm}$ is located at the position $(r, z)=(0.75 \mathrm{~m}, 0.51 \mathrm{~m})$ on the inner wall of the shell. The reference distributions of magnetic flux inside the shell and the eddy current on the shell were analysed beforehand using the RELAX-Fit code [4].

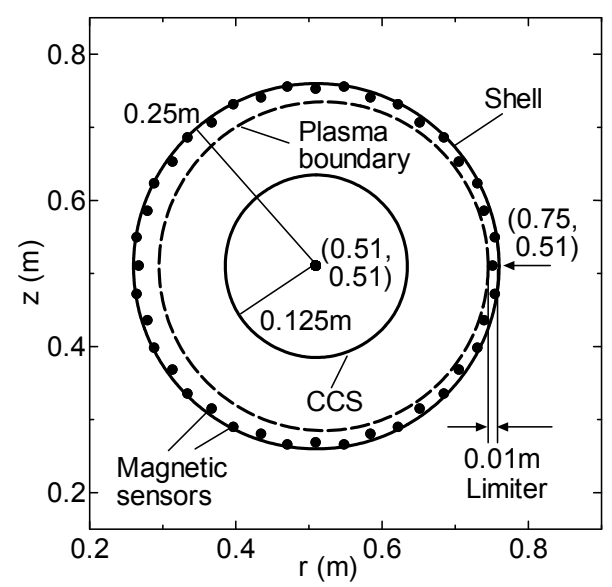

Figure 1: Image of the RELAX limiter configuration. 
The signals of magnetic sensors were also known before the present inverse analyses. The sensor locations are also illustrated in Figure 1. One assumes 40 sensor positions around two circles with a common centre at $(r, z)=(0.51 \mathrm{~m}$, $0.51 \mathrm{~m}$ ) inside the shell: 20 points are around the circle with a radius of $0.242 \mathrm{~m}$ at even intervals, while the other 20 are at a distance of $0.248 \mathrm{~m}$ from the centre. Each position is $8 \mathrm{~mm}$ and $2 \mathrm{~mm}$ away from the shell, respectively. One here hypothetically assumes that both a toroidal flux loop and a tangential probe are located at each of the 40 positions. That is, a total of 80 magnetic sensors are assumed. The tangential probe detects the magnetic field component that is tangential to the shell surface in the poloidal direction.

\section{Method}

In this section, one describes how the effect of the eddy current on the shell is incorporated into the original CCS method. The eddy current distribution on the shell, in the same way as all other physical quantities, is assumed to be axisymmetric in the toroidal direction.

\subsection{The set of boundary integral equations}

The Cauchy-condition surface (CCS), where both the Dirichlet and the Neumann conditions (i.e. the magnetic flux function $\psi$ and its normal derivative $\partial \psi / \partial n$ ) are unknown, is hypothetically placed in a domain that can be supposed to be inside the plasma. In the analysis, no plasma current is assumed outside this CCS, where in reality plasma current does exist. Instead, the CCS plays the same role as the plasma current in causing the field outside the plasma.

For an axisymmetric $(r, z)$ system, the differential form of Ampere's law $\mu_{0} \mathbf{j}=\nabla \times \mathbf{B}$ can be reduced to a partial differential equation

$$
-\Delta^{*} \psi \equiv-\left\{r \frac{\partial}{\partial r}\left(\frac{1}{r} \frac{\partial}{\partial r}\right)+\frac{\partial^{2}}{\partial z^{2}}\right\} \psi=\mu_{0} r\left(j_{\mathrm{Pl}}+j_{\text {Coil }}+j_{\mathrm{Eddy}}\right)
$$

in terms of magnetic flux function $\psi$. Here, $j_{\mathrm{Pl}}, j_{\text {Coil }}$, and $j_{\mathrm{Eddy}}$ denote the toroidal components of the plasma current, the external coil current and the eddy current respectively. The quantity $\mu_{0}$ is the permeability of a vacuum. In the following formulation one uses the magnetic flux $\tilde{\psi}=2 \pi \psi[\mathrm{Wb}]$ instead of the magnetic flux function $\psi[\mathrm{Wb} / \mathrm{rad}]$ because the physical quantity directly measured is the magnetic flux. Also, instead of the magnetic field signal $B$, a quantity $\tilde{B}=2 \pi B$ is defined.

To evaluate $\tilde{\psi}$ and $\partial \tilde{\psi} / \partial n$ at six points along the $\operatorname{CCS}\left(\Gamma_{\mathrm{CCS}}\right)$, three types of boundary integral equations (BIEs) can be given using the sensor signals and the external poloidal coil current data, as shown in the following. 
(i) For the magnetic 'flux' signal $\tilde{\psi}_{i}$ at points $i$ :

$$
\tilde{\psi}_{i}-\tilde{W}_{i}^{\psi}=\int_{\Gamma_{\mathrm{cCS}}}\left(\frac{\psi^{*}}{r} \frac{\partial \tilde{\psi}}{\partial n}-\frac{\tilde{\psi}}{r} \frac{\partial \psi^{*}}{\partial n}\right) d \Gamma+2 \pi \mu_{0} \int_{\Gamma_{\text {Shell }}} j_{S}\left(\mathbf{r}_{s}\right) \psi^{*}\left(\mathbf{r}_{s} \rightarrow \mathbf{r}_{i}\right) d \Gamma\left(\mathbf{r}_{s}\right) .
$$

(ii) For the magnetic 'field' signal $B_{i}$ at points $i$ :

$$
\tilde{B}_{i}-\tilde{W}_{i}^{B}=\int_{\Gamma_{\mathrm{cCS}}}\left(\frac{B^{*}}{r} \frac{\partial \tilde{\psi}}{\partial n}-\frac{\tilde{\psi}}{r} \frac{\partial B^{*}}{\partial n}\right) d \Gamma+2 \pi \mu_{0} \int_{\Gamma_{\text {Shell }}} j_{S}\left(\mathbf{r}_{s}\right) B^{*}\left(\mathbf{r}_{s} \rightarrow \mathbf{r}_{i}\right) d \Gamma\left(\mathbf{r}_{s}\right),
$$

using the quantity $\tilde{B}_{i}=2 \pi B_{i}$, where $B_{i}=-\mathbf{n}_{\mathbf{0}} \cdot \nabla \psi_{i} / r_{i}, B^{*}=-\mathbf{n}_{\mathbf{0}} \cdot \nabla \psi^{*} / r$ with $\mathbf{n}_{\mathbf{0}}$ being the assigned vector normal to the direction of the 'magnetic probe' located at the point $i$.

(iii) For points $i$ on the CCS:

$$
\frac{1}{2} \tilde{\psi}_{i}-\tilde{W}_{i}^{C}=\int_{\Gamma_{\mathrm{CCS}}}\left(\frac{\psi^{*}}{r} \frac{\partial \tilde{\psi}}{\partial n}-\frac{\tilde{\psi}}{r} \frac{\partial \psi^{*}}{\partial n}\right) d \Gamma+2 \pi \mu_{0} \int_{\Gamma_{\text {Shell }}} j_{S}\left(\mathbf{r}_{s}\right) \psi^{*}\left(\mathbf{r}_{s} \rightarrow \mathbf{r}_{i}\right) d \Gamma\left(\mathbf{r}_{s}\right)
$$

In eqns (2a)-(2c), $\tilde{W}_{i}^{\psi}, \tilde{W}_{i}^{B}$ and $\tilde{W}_{i}^{C}$ are the contributions of the external coil currents to the point $i$. In each equation, $\psi^{*}$ denotes the fundamental solution which satisfies a subsidiary equation

$$
-\left\{r \frac{\partial}{\partial r}\left(\frac{1}{r} \frac{\partial}{\partial r}\right)+\frac{\partial^{2}}{\partial z^{2}}\right\} \psi^{*}=r \delta_{i}
$$

where Dirac's delta function $\delta_{i}$ means $\delta(r-a) \delta(z-b)$ with the spike at the point $i$, where $i$ is defined as having the coordinates $(a, b)$. The detailed form of $\psi^{*}$ is given by $[1,2]$

$$
\psi^{*}=\frac{\sqrt{a r}}{\pi k}\left[\left(1-\frac{k^{2}}{2}\right) K(k)-E(k)\right]
$$

with

$$
k^{2}=\frac{4 a r}{(r+a)^{2}+(z-b)^{2}},
$$


where $K(k)$ and $E(k)$ are the complete elliptic integrals of the first and the second kind, respectively.

The second term on the RHS in each of eqns (2a), (2b) and (2c) describes the effect of the eddy current on the shell. In the quantity $\psi^{*}\left(\mathbf{r}_{s} \rightarrow \mathbf{r}_{i}\right), \mathbf{r}_{s}$ means an arbitrary point on the shell. The quantity $j_{S}\left(\mathbf{r}_{s}\right)$ denotes the linear density $[\mathrm{MA} / \mathrm{m}]$ distribution of the eddy current on the shell, which is integrated in the poloidal direction along the shell $\left(\Gamma_{\text {Shell }}\right)$.

\subsection{Accurate computation of boundary integrals along the shell}

The boundary integrals along the shell (the second term on the RHS in each of eqns (2a), (2b) and (2c)) should be performed very carefully. Since the distance $\varepsilon=\sqrt{(r-a)^{2}+(z-b)^{2}}$ between the sensor position $(a, b)$ and an integration point $(r, z)$ on the shell is very short, the following singularities [5]

$$
\begin{gathered}
\psi^{*} \rightarrow-\frac{a}{2 \pi} \log \varepsilon, \\
\frac{\partial \psi^{*}}{\partial a} \rightarrow-\frac{1}{4 \pi} \log \varepsilon+\frac{a(r-a)}{2 \pi} \cdot \frac{1}{\varepsilon^{2}}
\end{gathered}
$$

and

$$
\frac{\partial \psi^{*}}{\partial b} \rightarrow \frac{a(z-b)}{2 \pi} \cdot \frac{1}{\varepsilon^{2}}
$$

arise in the integration kernels when $\varepsilon \rightarrow 0$.

In a boundary element $\left(\Gamma_{\text {Shell, }, j}\right)$ along the shell, one here uses the notations $G(\xi)$ and $\phi(\xi)$ for the Jacobian of the coordinate transformation and one of the interpolation functions, respectively. Suppose that $B^{*}(\xi)$ is the fundamental solution or its derivative, while $F_{S}(\xi)$ denotes the corresponding asymptotic function, i.e. eqns (6), (7) or (8). The general form of the boundary integral over $\Gamma_{\text {Shell, } j}$ can be rearranged as

$$
\int_{-1}^{1} \phi(\xi) G(\xi) B^{*}(\xi) d \xi=\int_{-1}^{1}\left\{\phi(\xi) G(\xi) B^{*}(\xi)-\phi_{0} G_{0} F_{S}(\xi)\right\} d \xi+\phi_{0} G_{0} \int_{-1}^{1} F_{S}(\xi) d \xi
$$

where $G_{0}$ and $\phi_{0}$ are the values of $G(\xi)$ and $\phi(\xi)$ at the position $\xi=\xi_{0}$ on the boundary element that is the nearest to the location of the magnetic sensor $i$ under consideration. The asymptotic function is subtracted from the original integrand in the first integral on the RHS of eqn (9), and this subtraction is compensated by 
the analytical integral of the second integral on the RHS. The total integrand of the first integral has no singularity and can therefore be evaluated with the ordinary Gaussian quadrature with 16 integration points for each boundary element.

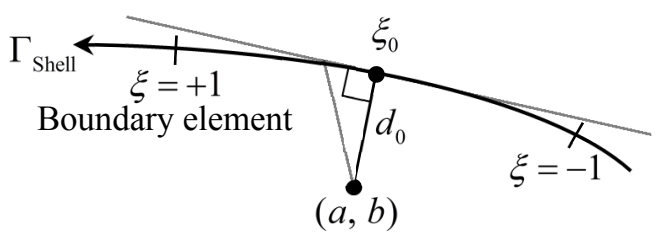

Sensor

position

Figure 2: The minimum distance ${ }^{d_{0}}$ from the sensor position $(a, b)$ to a boundary element.

Ma and Kamiya [6] proposed the use of an approximated 'distance function' for the boundary element adjacent to the sensor position $(a, b)$ as

$$
d(\xi)=G_{0} \sqrt{\left(\xi-\xi_{0}\right)^{2}+\left(d_{0} / G_{0}\right)^{2}},
$$

where $d_{0}$ is the minimum distance from the point $(a, b)$ to the boundary element as shown in Figure 2, which corresponds to the local coordinate $\xi=\xi_{0}$. Equation (10) agrees with $\varepsilon$ in eqns (6), (7) and (8) when $\xi \rightarrow \xi_{0}$ and $d_{0} \rightarrow 0$.

The quantities in eqns (7) and (8) can be rewritten in the following forms:

$$
\frac{a(r-a)}{2 \pi} \cdot \frac{1}{\varepsilon^{2}}=\frac{a}{2 \pi G_{0}^{2}}\left[\alpha_{0}+\frac{\alpha_{1}\left(\xi-\xi_{0}\right)}{\left(\xi-\xi_{0}\right)^{2}+\left(d_{0} / G_{0}\right)^{2}}+\frac{\alpha_{2}}{\left(\xi-\xi_{0}\right)^{2}+\left(d_{0} / G_{0}\right)^{2}}\right]
$$

and

$$
\frac{a(z-b)}{2 \pi} \cdot \frac{1}{\varepsilon^{2}}=\frac{a}{2 \pi G_{0}^{2}}\left[\beta_{0}+\frac{\beta_{1}\left(\xi-\xi_{0}\right)}{\left(\xi-\xi_{0}\right)^{2}+\left(d_{0} / G_{0}\right)^{2}}+\frac{\beta_{2}}{\left(\xi-\xi_{0}\right)^{2}+\left(d_{0} / G_{0}\right)^{2}}\right] .
$$

The terms having singularities can be integrated analytically, e.g.

$$
\int_{-1}^{1} \frac{\left(\xi-\xi_{0}\right)}{\left(\xi-\xi_{0}\right)^{2}+\left(d_{0} / G_{0}\right)^{2}} d \xi=\log \sqrt{\left(1-\xi_{0}\right)^{2}+\left(\frac{d_{0}}{G_{0}}\right)^{2}}-\log \sqrt{\left(1+\xi_{0}\right)^{2}+\left(\frac{d_{0}}{G_{0}}\right)^{2}} \text {. }
$$


Figure 3 compares the integrands in terms of $\partial \psi^{*} / \partial b$ with an interpolation function $\phi_{2}=(1-\xi)(1+\xi)$ before and after damping out the singularity for a case where 10 boundary elements are used along the shell. The strong singularity is efficiently damped out. As the modified integrand in Figure 3(b) still has a small edge at $\xi_{0}=0.5$, the first integral on the RHS of eqn (9) is divided into two integral intervals, $\left[-1, \xi_{0}\right]$ and $\left[\xi_{0},+1\right]$ as long as $\xi_{0} \neq \pm 1$.

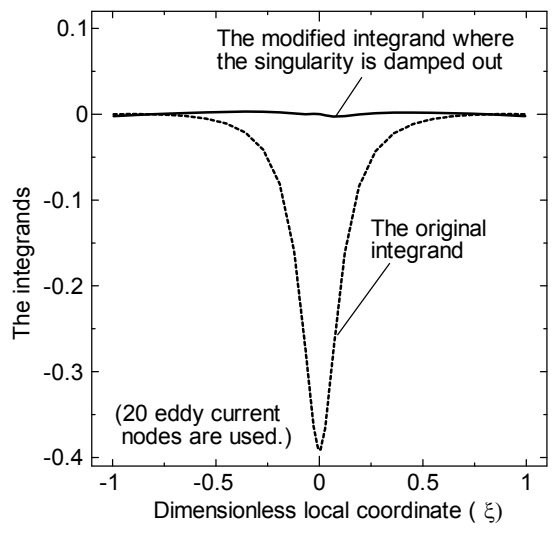

(a) $\xi_{0}=0.0, d_{0}=8 \mathrm{~mm}$

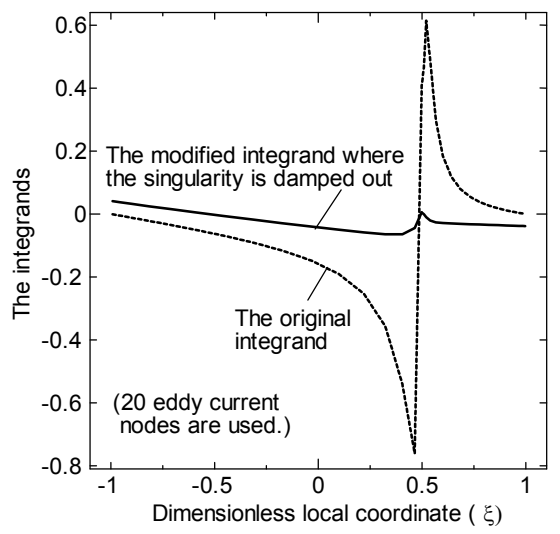

(b) $\xi_{0}=0.5, d_{0}=2 \mathrm{~mm}$

Figure 3: Integrands before and after damping out the singularity.

\subsection{Discretization}

Using the quadratic boundary elements, eqns (2a), (2b) and (2c) are discretized, coupled and can be expressed in a matrix form

$$
\mathbf{D p}=\mathbf{g},
$$

where $\mathbf{D}$ is an $m \times n$ matrix. The solution vector $\mathbf{p}$ contains $N_{S}$ nodes of linear D eddy current density on the shell as well as the set of $N_{C}$ flux values and their $N_{C}$ normal derivatives on the CCS. Then, the total number of unknowns is $n=2 N_{C}+N_{S}$, while the number of BIEs is given by $m=N_{\psi}+N_{B}+N_{C}$ with $N_{\psi}$ and $N_{B}$ being the numbers of flux loops and field sensors, respectively. The quantities $\psi_{i}-W_{i}^{\psi}$ and $B_{i}-W_{i}^{B}$ are stored in the vector $\mathbf{g}$ in eqn (14).

The matrix equation (14) is solved using the singular value decomposition (SVD) technique [7]. The matrix $\mathbf{D}$ is decomposed as $\mathbf{D}=\mathbf{U} \mathbf{\Lambda} \mathbf{V}^{\mathrm{T}}$, where $\mathbf{U}$ and $\mathbf{V}^{\mathrm{T}}$ are orthogonal matrices and $\boldsymbol{\Lambda}$ is a diagonal matrix with positive singular values or zero components. In the so-called truncated SVD (TSVD) technique [7], the regularized solution is given by 


$$
\mathbf{p}_{k}=\mathbf{V} \boldsymbol{\Lambda}_{k}^{-1} \mathbf{U}^{\mathrm{T}} \mathbf{g}
$$

Here $\boldsymbol{\Lambda}_{k}$ means that the singular values smaller than $\lambda_{k}$ in $\boldsymbol{\Lambda}$ are omitted so that the condition number is not larger than a certain value.

Once all the values of the Cauchy conditions on the CCS and the linear eddy current densities on the shell nodes are known, the magnetic flux $\tilde{\psi}_{i}$ for arbitrary points outside the CCS can be calculated using eqn (2a).

\subsection{Solving an ill-conditioned matrix equation - the MTSVD method}

Figure 4 shows the behaviour of the singular values which appeared in the SVD process for various numbers of assumed eddy current nodes, $N_{s}$. The vertical axis represents the singular values whose maximum value is normalized to unity. The smallest singular value becomes less than $10^{-3}$ when $N_{S}$ is greater than 40 .

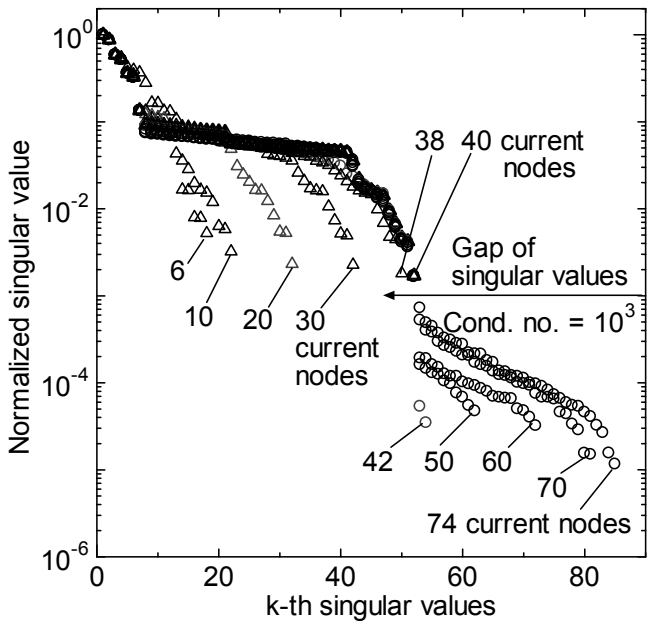

Figure 4: The singular values with the number of eddy current nodes.

A gap is commonly observed in the vicinity of $10^{-3}$ of the normalized singular values. When the number of current nodes $N_{S}$ exceeds 40 , the condition number (the ratio of the largest to the smallest singular values; the reciprocal of the normalized singular value) jumps up to over $10^{4}$. If one truncates the singular values smaller than the gap threshold observed in Figure 4, the improved condition numbers are around $6 \times 10^{2}$ in all cases.

Even with this TSVD technique, however, a numerical oscillation of $\mathbf{p}_{k}$ is observed when the smallest singular value is smaller than the gap threshold. One idea to suppress such an oscillation is to introduce a constraint, $\min \|\mathbf{L} \mathbf{p}\|$, in 
addition to $\min \|\mathbf{D} \mathbf{p}-\mathbf{g}\|$, where $\mathbf{L}$ means a differential operator. Based on this idea, Hansen et al. [8] derived the modified TSVD (MTSVD) solution

$$
\mathbf{p}_{L, k}=\mathbf{p}_{k}-\mathbf{V}_{k}\left(\mathbf{L} \mathbf{V}_{k}\right)^{+} \mathbf{L} \mathbf{p}_{k}=\mathbf{p}_{k}-\mathbf{V}_{k} \mathbf{z}_{k}
$$

where $\mathbf{V}_{k}=\left[V_{k+1}, \cdots, V_{n}\right]$ and $\mathbf{z}_{k}$ is the solution of

$$
\left(\mathbf{L} \mathbf{V}_{k}\right) \mathbf{z}_{k}=\mathbf{L p}_{k}
$$

One uses the following $(n-6) \times n$ matrix for a total of $n$ unknowns:

$$
\mathbf{L}=\left[\begin{array}{ccc}
\mathbf{L}^{(\mathrm{N})} & \mathbf{0} & \mathbf{0} \\
\mathbf{0} & \mathbf{L}^{(\mathrm{D})} & \mathbf{0} \\
\mathbf{0} & \mathbf{0} & \mathbf{L}^{(\mathbf{E})}
\end{array}\right]
$$

In eqn $(18), \mathbf{L}^{(\mathrm{N})}, \mathbf{L}^{(\mathrm{D})}$ and $\mathbf{L}^{(\mathrm{E})}$ are discrete approximations to the second derivative operator applied to the Neumann conditions on the CCS, the Dirichlet conditions on the CCS and the current density solution on the shell surface, respectively, each of which has the tridiagonal form

$$
\mathbf{L}^{(\mathrm{E})}=\left[\begin{array}{cccccc}
1 & -2 & 1 & & & \\
& 1 & -2 & 1 & & \\
& & \ddots & \ddots & \ddots & \\
& & & 1 & -2 & 1
\end{array}\right] .
$$

to obtain a smooth solution.

Figure 5 illustrates the effectiveness of the MTSVD method when applied to a test case where 60 eddy current nodes are assumed. The solid grey curve that oscillates at high frequency is the original solution $\left(\mathbf{p}_{k}\right)$ that is produced using the ordinary TSVD technique. The number of peaks in this curve, 60, agrees with the number of assumed eddy current nodes. The dotted curve depicts the correction vector that is given by $\mathbf{V}_{k} \mathbf{z}_{k}$ in eqn (16). Subtracting the dotted curve from the grey curve, i.e. following eqn (16), one obtains the solid black curve $\left(\mathbf{p}_{L, k}\right)$ where the numerical oscillation has been drastically damped out. Although there are slight ripples in the resultant curve, it is worth mentioning that the number of ripples agrees not with the number of current nodes any more but with the number of sensor locations $(=40)$, which are the singular points, $i$, indicated in eqns $(2 \mathrm{a})$ and $(2 b)$. 


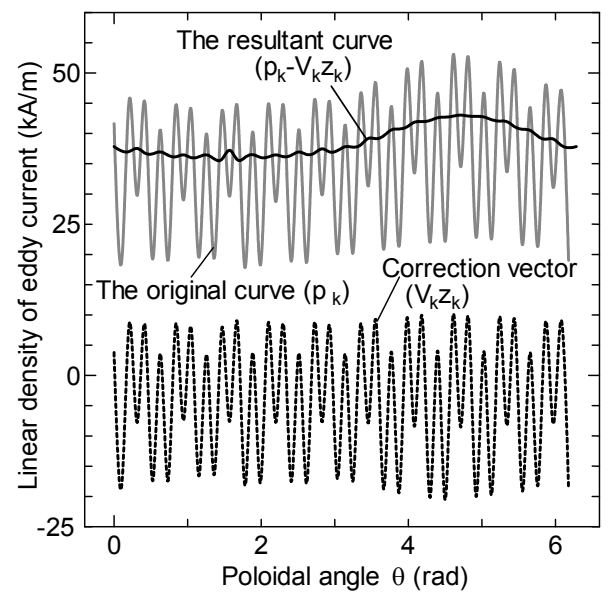

Figure 5: Subtraction of correction vector from the original solution.

\section{Numerical demonstration for the RELAX device}

In the present work the CCS approximates a circle having a radius of $0.125 \mathrm{~m}$ and centre $(r, z)=(0.51 \mathrm{~m}, 0.51 \mathrm{~m})$, as also shown in Figure 1 . The circle is divided into 3 continuous quadratic boundary elements, so that the total number of nodes is 6 (the number of unknowns on the CCS becomes 12).

\subsection{Reconstructed profiles of eddy current density and magnetic flux}

Figures 6(a)-(d) show the variation in the eddy current density on the shell surface for the cases assuming 20,30, 40 and 60 eddy current nodes, respectively. In each figure the vertical axis denotes the current density, while the abscissa means the poloidal angle $\theta$ that varies in the clockwise direction whose starting point $(\theta=0)$ on the shell is at the top $(r, z)=(0.51 \mathrm{~m}, 0.76 \mathrm{~m})$. The dashed and the solid curves in Figure 6 denote the reference and the reconstructed variation in the eddy current density, respectively.

Note here that the MTSVD technique is applied in cases where one truncates the singular values smaller than the gap threshold shown in Figure 4, i.e. the cases where the number of current nodes is greater than 40. That is, Figure 6(d) is the result when using the MTSVD technique.

Figures 7(a) and 7(b) show the reconstructed flux profiles for the 40 and 60 current node cases. In each figure the dashed contours show the reference solution, while the solid contours indicate the reconstructed flux. In an ordinary CCS method analysis, the reconstructed flux solution is inaccurate in the domain where the plasma current exists. However, in the present RELAX analyses, accurate reconstructions can be observed even deep inside the plasma region. This is because the eddy current effect is dominant over the plasma current effect for the formation of the flux distribution in the RELAX device. 


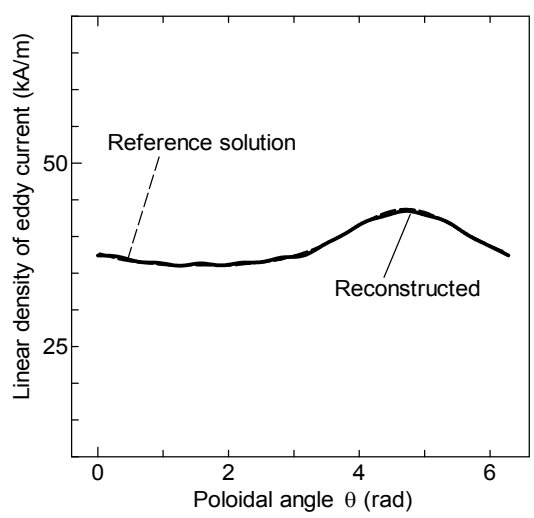

(a) 20 current nodes

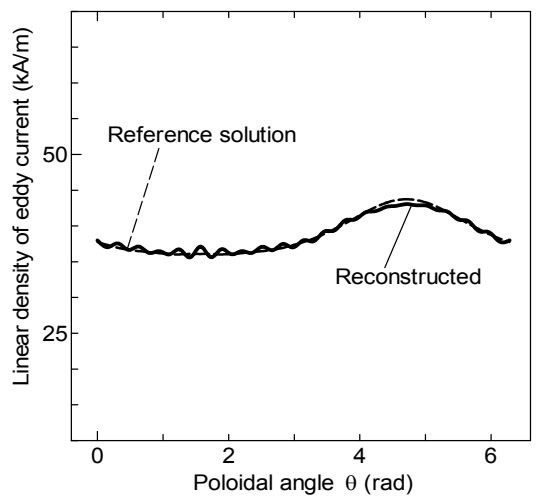

(c) 40 current nodes

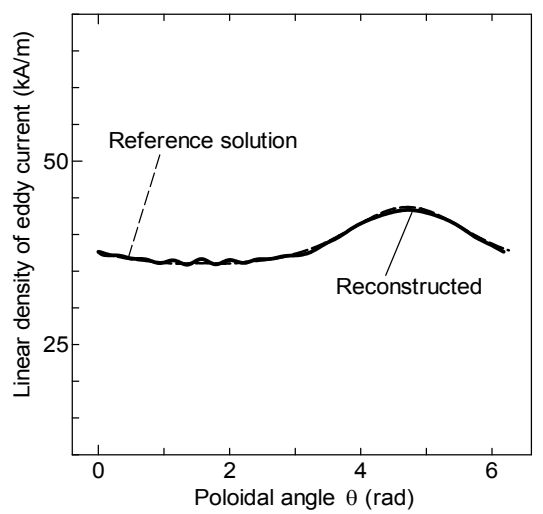

(b) 30 current nodes

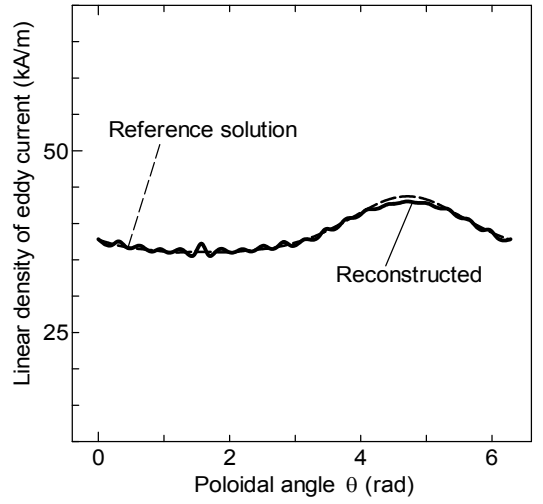

(d) 60 current nodes

Figure 6: Reconstruction of the eddy current profile.

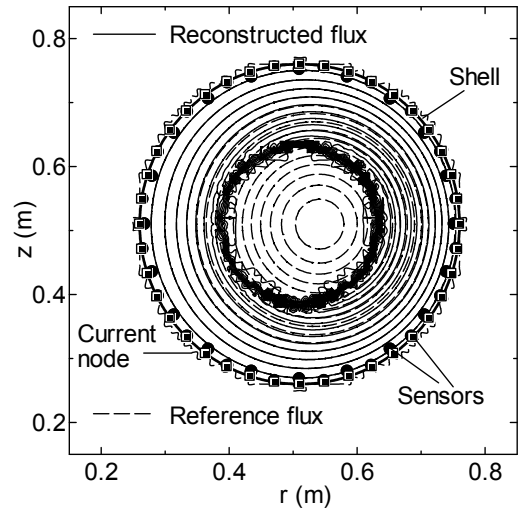

(a) 40 current nodes

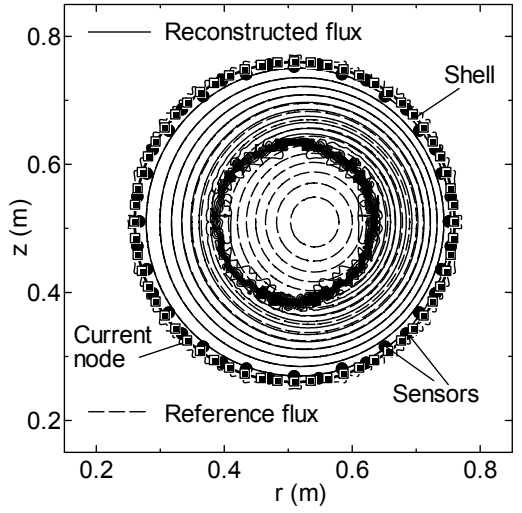

(b) 60 current nodes

Figure 7: Reconstruction of the magnetic flux profile. 


\subsection{Influence of the sensor signal noise}

The effect of measurement errors on the reconstruction was also studied. Noise was numerically generated and added to all field and flux loop signals. A noise-added signal is given by $\tilde{b}_{j}=b_{j}(1+\sigma \cdot G)$, where $b_{j}$ is the original signal, $G$ denotes a unit Gaussian random number, and $\sigma$ is the standard deviation of the Gaussian noise.

Without sensor signal noise, the reconstructed current density profile shown in Figure 6(a) under the adoption of 20 current nodes seems to be in good agreement with the reference profile. However, it is premature to make a conclusion that the best choice is this number of current nodes. Figure 8 shows the relative errors of reconstructed flux and eddy current density as functions of the number of current nodes under the assumption of $3 \% \sigma$ noise. Unfortunately the solutions are sensitive to the signal noise if the number of current nodes is less than 40 .

In Figure 8, the dashed curve indicates the variation in the condition number multiplied by $3 \%$, which is the theoretical maximum of the error caused by the $3 \%$ $\sigma$ noise. (The condition numbers with over 40 current nodes mean the results after truncating the small singular values.) It should be noticed that, in spite of the comparatively small condition numbers, the observed errors with fewer than 40 nodes are much larger than those with over 40 nodes. Accordingly, this phenomenon cannot be explained by the magnitude of the condition number. Rather, it is suggested that this is caused by the lack of information as a constraint in the inverse analysis. One should adopt a number of current nodes which is large enough to ensure that all singular values larger than the gap threshold, which have meaningful physical information, are taken into account in the analysis for obtaining a robust solution.

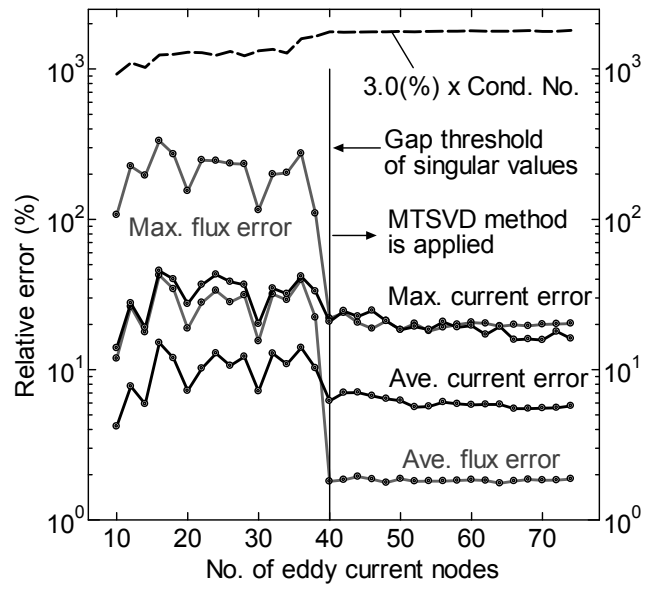

Figure 8: Influence of the sensor signal noise when $3 \% \sigma$ noise is imposed. 


\section{Conclusion}

(1) As the sensors are closely adjacent to the shell, the near singular boundary integrals along the shell should be accurately evaluated. This singularity is damped out effectively with the algorithm based on the approximated distance function.

(2) If the smallest singular value is smaller than the gap threshold, a numerical oscillation of the eddy current profile is observed. This oscillation is eliminated effectively by applying the MTSVD technique.

(3) To obtain a solution stable against sensor signal noise, one should adopt enough number of eddy current nodes to ensure that all singular values larger than the gap threshold are taken into account.

The present techniques are applicable to the problem of eddy current flow in a conductor located close to a magnetic sensor in many other devices.

\section{Acknowledgements}

This research was performed with the support and under the auspices of the NIFS Collaboration Research Program (NIFS12KLPP024 \& NIFS12KNWP002), also supported by the Ministry of Education, Culture, Sports, Science and Technology, Grant-in-Aid for Scientific Research (C), 24561019, 2012.

\section{References}

[1] Kurihara, K., Fusion Eng. Des. 51-52 1049, 2000.

[2] Itagaki, M., Yamaguchi, S. \& Fukunaga, T., Nucl. Fusion 45, 153-162, 2005.

[3] Masamune, S., Sanpei, A., Ikezono, R., Onchi, T., Oki, K, Yamashita, T., Shimazu, H., Himura, H. \& Paccagnella, R., J. Fusion Energ. 28, 187, 2009.

[4] Sanpei, A., Oki, K., Ikezoe, R., Onchi, T., Murata, K., Shimazu, H., Yamashita, T., Fujita, S., Himura, H., Masamune, S. \& Anderson, J. K., J. Phys. Soc. Jpn. 78[1], 013501, 2009.

[5] Itagaki, M. \& Shimoda, H., Eng. Anal. Boundary Elements 33, 845, 2009.

[6] Ma, H. \& Kamiya, N., Eng. Anal. Boundary Elements 25, 833, 2001.

[7] Hansen, P.C., Rank-Deficient and Discrete Ill-Posed Problems - Numerical Aspects of Linear Inversion (Philadelphia, SIAM, 1998).

[8] Hansen, P.C., Sekii, T. \& Shibahashi, H., SIAM J. Sci. Stat. Comput., 13, 1142, 1992. 